\section{Rsal, un ARN régulateur aux multiples facettes, module le métabolisme du pathogène opportuniste Staphylococcus aureus}

Emma Desgranges ${ }^{1}$, Delphine Bronesky ${ }^{1 *}$, Anna Corvaglia ${ }^{2}$, Patrice François ${ }^{2}$, Carlos Caballero ${ }^{3}$, Laura Prado ${ }^{3}$, Alejandro Toledo-Arana ${ }^{3}$, Inigo Lasa ${ }^{4}$, Karen Moreau ${ }^{5}$, François Vandenesch ${ }^{5}$, Stefano Marzi ${ }^{1}$, Pascale Romby ${ }^{1}$, Isabelle Caldelari ${ }^{1}$
${ }^{1}$ Université de Strasbourg, CNRS, Architecture et réactivité de l'ARN, UPR9002, F-67000 Strasbourg, France.

${ }^{2}$ Laboratoire de recherche en génomique, département de spécialités médicales, Genève hôpitaux universitaires, université de Genève, Genève, Suisse.

${ }^{3}$ Instituto de agrobiotecnología. CSIC-UPNA-GN, 31192-Mutilva, Navarra, Espagne.

${ }^{4}$ Navarrabiomed, universidad pública de Navarra, departamento de salud, IDISNA, Pamplona, Espagne.

${ }^{5} \mathrm{CIRI}$, centre international de recherche en infectiologie, Inserm U1111, université Lyonl, école normale supérieure de Lyon, CNRS UMR5308, F-69008 Lyon, France.

*Adresse actuelle: ARNA, U1212, UMR 5320, université de Bordeaux, 146 rue Leo Saignat, Carreire zone nord Bat 3A $1^{\text {er }}$, BP12, 33076 Bordeaux, France.

i.caldelari@unistra.fr
> Staphylococcus aureus est une bactérie commensale retrouvée chez environ $30 \%$ des individus sains dont elle colonise la peau et la muqueuse nasale. Cependant, c'est également une bactérie pathogène opportuniste responsable d'infections diverses telles que orgelet, ostéomyélite, endocardite, ou encore septicémie en envahissant un grand nombre de tissus et d'organes. Cette bactérie est capable de s'adapter à des conditions hostiles et variées, telles que carence nutritive et stress osmotique, oxydant, ou thermique, ainsi qu'à la réponse immunitaire de I'hôte, car elle produit une grande diversité de facteurs de virulence. La synthèse de ces facteurs est finement régulée par des protéines et des ARN régulateurs majoritairement non codants, souvent désignés par l'abréviation sARN (dérivée de l'anglais, small RNA). Les facteurs de transcription et les systèmes à deux composants contrôlent l'expression des gènes impliqués non seulement dans le métabolisme, mais aussi dans la réponse au stress et la virulence [1]. Par exemple, la protéine du contrôle catabolique (carbon catabolite control protein $A,(c p A)$ a un rôle essentiel dans le choix de la source carbonée en régulant le métabolisme central de la bactérie ainsi que la virulence $[2,3]$. CcpA se fixe à une séquence promotrice spécifique appelée cre (catabolite-responsive element), qui est très conservée chez les bactéries à Gram positif [2]. Quant aux sARN, ils interagissent principalement avec leurs ARN messagers (ARNm) cibles. L'hybridation peut conduire à la stabilisation/ déstabilisation de I'ARNm ou à l'activation/répression de sa traduction [4].

Nous avons montré que la transcription du sARN Rsal (RNA Staphylococcus aureus I) est réprimée par (cpA en présence de glucose [5]. L'induction de la synthèse de Rsal signale que la concentration en glucose diminue dans le milieu extracellulaire et que la croissance des bactéries est ralentie. $\varepsilon$ n interagissant avec ses ARNm cibles ou d'autres SARN, il permet à la population bactérienne de modifier son métabolisme lorsque la source carbonée primaire est consommée.

\section{Rsal est inhibé par CcpA en présence de glucose}

Rsal est un SARN de 144 nucléotides, très conservé chez les staphylococcaceae, et qui comporte deux motifs riches en guanosine ainsi qu'une longue région simple brin riche en pyrimidines. Un motif cre de liaison à CcpA a été détecté dans la région promotrice du gène codant Rsal. Ce sARN est exprimé dès l'entrée en phase stationnaire de croissance des bactéries dans un milieu riche contenant $2 \%$ de glucose [6]. Néanmoins, dans un milieu sans glucose ou dans une souche délétée du gène ccpA, Rsal est exprimé de manière constitutive, suggérant que CcpA est le principal répresseur de Rsal en présence de glucose et que cette répression est levée après consommation du glucose (en début de phase stationnaire).

\section{L’identification des cibles de Rsal révèle ses fonctions}

La technique MAPS (MS2-affinity purification coupled with RNA sequencing) a été utilisée afin d'identifier l'ensemble des cibles ARN directes de Rsal (Figure 1) [7]. Cette technique permet d'isoler les complexes formés entre Rsal et ses ARN associés in vivo. Rsal a été au préalable fusionné à une étiquette MS2 placée en 5' du sARN. Cette étiquette est spécifiquement reconnue par la protéine de capside du phage MS2 (MS2-CP), qui est elle-même fixée sur une résine d'amylose pour retenir spécifiquement les complexes contenant Rsal-MS2. En couplant à cette chromatographie d'affinité une technique de séquençage à haut débit (Miseq, Illumina), les ARN liés à RsalMS2 sont caractérisés. L'interaction directe entre Rsal et ses cibles potentielles, ainsi que les conséquences de son action sur la régulation du métabolisme de la bactérie ont été ensuite validées par une combinaison d'approches in 


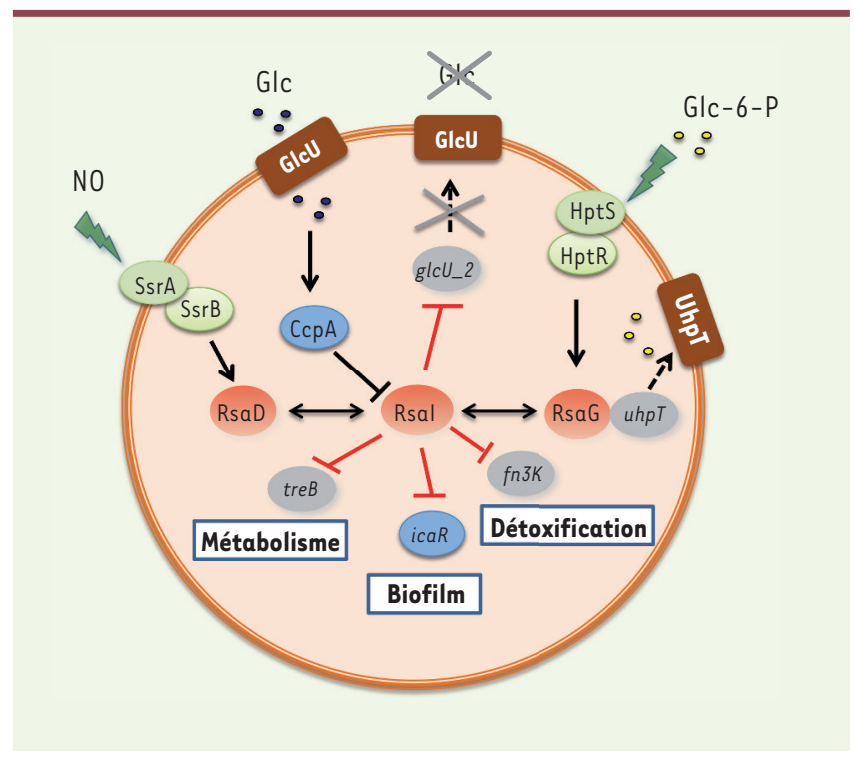

Figure 1. Représentation schématique des réseaux de régulation centrés sur l'ARN régulateur Rsal. Les SARN sont colorés en rouge, les facteurs de transcription en bleu, les systèmes à deux composants en vert, les ARNm en gris, et les transporteurs en marron. Les connecteurs noirs ou rouges indiquent respectivement un contrôle transcriptionnel ou post-transcriptionnel. Les flèches symbolisent une activation, tandis que les barres transversales indiquent une inhibition. sARN : small RNA; Rsal : RNA Staphylococcus aureus I.

la déglycation des protéines en présence de fortes concentrations de glucose (Figure 2A). Les deux motifs riches en guanine vivo et in vitro. Ainsi, nous avons révélé l'existence de deux classes de cibles pour Rsal : des ARNm impliqués dans l'importation du glucose ( $\left.g l c U \_2\right)$, le métabolisme des sucres (treB), la détoxification des protéines $(f n 3 K)$, et la formation des biofilms (icaR), et des sARN possédant des séquences riches en cytosine, complémentaires de la séquence du site de fixation du ribosome sur leurs ARNm cibles (séquence de Shine-Dalgarno). Par ailleurs, le transcriptome différentiel indique que Rsal inhiberait la voie des pentoses phosphate et, à l'inverse, activerait des enzymes des voies de fermentation ainsi que d'autres protéines requises pour résister au monoxyde d'azote (NO), un métabolite libéré par les cellules endothéliales, les polynucléaires neutrophiles, et les macrophages de l'hôte.

\section{Rsal contient deux domaines fonctionnels distincts}

La mutagenèse dirigée a permis de distinguer deux motifs fonctionnels de Rsal. Sa région en simple brin riche en adénine et uridine interagit avec les séquences de Shine-Dalgarno de ses ARNm cibles, inhibant ainsi leur traduction. C'est le cas pour les transcrits codant le transporteur du glucose GIcU_2, le transporteur du tréhalose TreB, et la fructosamine kinase $\mathrm{Fn} 3 \mathrm{~K}$ impliquée dans interagissent quant à eux avec les motifs riches en cytosine des sRNA RsaG et RsaD. RsaG est induit par la présence de glucose-6-phosphate (G6P) dans le milieu extracellulaire sous le contrôle du système à deux composants HptRS (hexose phosphate transport RS, $R$ désignant le régulateur et $S$ le senseur), alors que RsaD répond au NO sous la dépendance du système SsrAB (staphylococcal respiratory response $A B$ ) (Figure l). Etonnamment, RsaG serait co-transcrit avec l'ARNm de uhpT (uptake of hexose phosphate transporter) codant le transporteur du G6P (Figure 1). Nous avons montré que la fixation de RsaG n'affecte pas les fonctions régulatrices de Rsal, telles que la répression traductionnelle de glcU_2 (Figures $I$ et $2 B$ ). II reste à déterminer si Rsal peut agir comme une « éponge à sARN » afin d'empêcher leur action.

Rsal module la synthèse de PIA-PNAG, un composant de la matrice des biofilms

S. aureus est la cause principale des infections nosocomiales chez les patients opérés pour la pose d'une prothèse, où se développent facilement des biofilms bactériens résistants au traitement antibiotique. L'opéron icaADBC code les enzymes responsables de la synthèse de l'exopolysaccharide PIA-PNAG (polysac- charide intercellular adhesin-poly-Nacetylglucosamine), composant majeur de la matrice des biofilms. Ces gènes sont réprimés par le facteur de transcription IcaR. L'ARNm icaR a la particularité d'inhiber sa propre traduction en induisant sa circularisation. En effet, une séquence riche en cytosine (antiSD) située dans la région 3' non-traduite (3'UTR pour 3' untranslated region) s'hybride à la séquence SD pour empêcher la fixation du ribosome et induire la dégradation de l'ARNm icaR par l'endoribonucléase III (Figure 2C) [8]. L'ARNm icaR a été identifié comme une cible de Rsal par la technique MAPS. Nous avons montré que Rsal interagit avec la 3'UTR de I'ARNm icaR en aval de la séquence anti-SD (Figure 2C). Des expériences permettant d'évaluer la production de PIA-PNAG indiquent que Rsal et la région 3'UTR de I'ARNm icaR sont nécessaires pour inhiber le répresseur IcaR et activer la synthèse de PIA-PNAG (Figure 2C).

\section{Conclusion}

Ces résultats illustrent le lien étroit entre la régulation du métabolisme, l'adaptation au stress, et la formation de biofilms bactériens chez $S$. aureus, impliquant le facteur de transcription CcpA et le sARN Rsal, et l'utilisation des sources carbonées. Cet ARN non-codant aux multiples facettes pourrait être principalement produit au niveau des sites d'inflammation où la concentration en glucose est faible (e.g., poumons, reins), et intervenir dans la persistance des bactéries lorsque les conditions sont défavorables à leur multiplication. II reste encore beaucoup à apprendre sur le rôle des SARN et sur leurs réseaux 
A

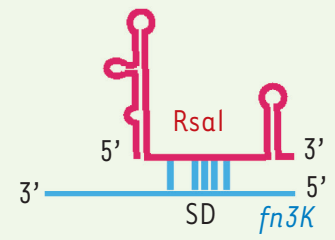

C

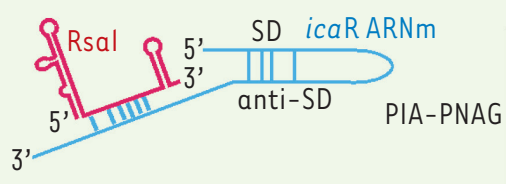

B

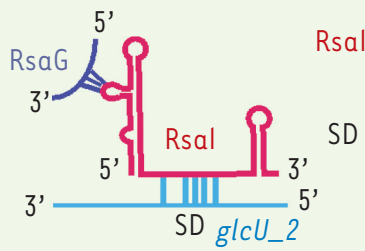

Rsal

$S D$

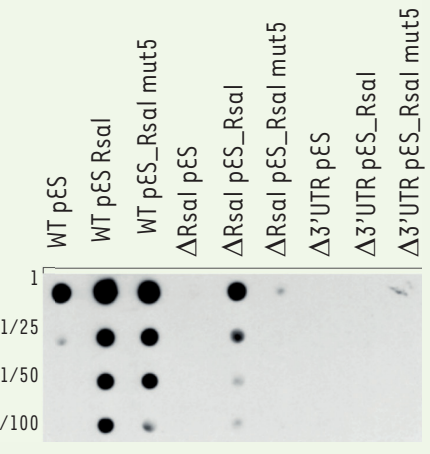

Figure 2. Divers mécanismes de régulation impliquant Rsal. A. Schéma du complexe formé entre Rsal et I'ARNm de fn3K. Rsal inhibe la traduction de cet ARNm en se fixant à sa séquence de Shine-Dalgarno (SD). B. Schéma du complexe formé entre Rsal, I'ARNm de glcU_2, et RsaG. La liaison de RsaG n'empêche pas Rsal de se fixer à son ARNm cible et d'y exercer son contrôle. C. Effet de Rsal sur la synthèse des exopolysaccharides PIA-PNAG in vivo. À gauche, schéma représentant Rsal se liant à la région 3' de l'ARNm icaR. L'interaction entre les séquences complémentaires SD et anti-SD est également indiquée. A droite, la biosynthèse de PIA-PNAG a été quantifiée par la technique de dot blot. Des échantillons ayant subi une série de dilutions (jusqu'à 1:100) ont été déposés sur une membrane de nitrocellulose, et la production de PIA-PNAG a été détectée avec des anticorps

spécifiques de cet exopolysaccharide dans la souche 132 de S. aureus (WT, pour wild-type), la souche délétée pour le gène rsal ( $\Delta$ rsal), et la souche portant une délétion de la région icaR J'UTR ( $\triangle 3^{\prime} U T R$ ). Ces trois souches ont été transformées soit avec un plasmide pES (pES), soit avec le plasmide recombinant exprimant rsal (pES_Rsal) ou sa forme mutée rsal mut5 (pES_Rsal mut5), dont la séquence d'interaction avec l'ARNm icaR a été inactivée par substitution (d'après [5]).

d'interactions dans le contrôle des voies métaboliques essentielles à l'invasion, la survie, et la dormance de S. aureus. Elucider ces mécanismes complexes de régulation permettra de mieux comprendre la pathogénicité de $S$. aureus, et de développer de nouvelles stratégies thérapeutiques contre les infections redoutables dont elle est parfois responsable. $\diamond$

Rsal, a multifaceted regulatory RNA, modulates the metabolism of the opportunistic pathogen Staphylococcus aureus

\section{LIENS D'INTÉRÊT}

Les auteurs déclarent n'avoir aucun lien d'intérêt concernant les données publiées dans cet article.

\section{RÉFÉRENCES}

1. Richardson AR, Somerville GA, Sonenshein AL. Regulating the intersection of metabolism and pathogenesis in Gram-positive bacteria. Microbiol Spectr 2015 ; 3 : 1-27.

2. Seidl K, Stucki M, Ruegg M, et al. Staphylococcus aureus $C \mathrm{cpA}$ affects virulence determinant production and antibiotic resistance. Antimicrob Agents Chemother 2006 ; 50 : 1183-94.

3. Li C, Sun F, Cho H, et al. CcpA mediates proline auxotrophy and is required for Staphylococcus aureus pathogenesis. J Bacteriol $2010 ; 192: 3883-92$.

4. Wagner $\varepsilon G H$, Romby P. Small RNAs in bacteria and archaea: who they are, what they do, and how they do it. Adv Genet $2015 ; 90: 133-208$.
5. Bronesky D, Desgranges $\varepsilon$, Corvaglia A, et al. A multifaceted small RNA modulates gene expression upon glucose limitation in Staphylococcus aureus. EMBO J 2019 ; 38 : e99363.

6. Geissmann T, Chevalier C, Cros M-J, et al. A search for small noncoding RNAs in Staphylococcus aureus reveals a conserved sequence motif for regulation. Nucleic Acids Res 2009; 37 : 7239-57.

7. Lalaouna D, Desgranges $\varepsilon$, Caldelari I, et al. MS2affinity purification coupled with RNA sequencing approach in the human pathogen Staphylococcus aureus. Meth Enzymol 2018 ; 612 : 393-411.

8. Ruiz de los Mozos I, Vergara-Irigaray M, Segura V, et al. Base pairing interaction between 5'- and 3'-UTRs controls icaR mRNA translation in Staphylococcus aureus. PLoS Genet 2013 ; 9 : e1004001.

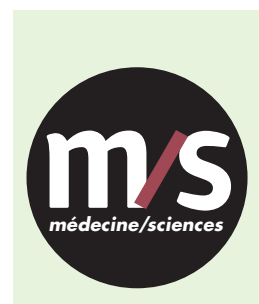

\section{Tarifs d'abonnement m/s - 2019 \\ Abonnez-vous \\ à médecine/sciences}

$>$ Grâce à $m / s$, vivez en direct les progrès des sciences biologiques et médicales

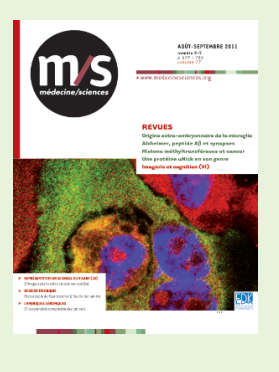

